OPEN ACCESS

Edited by:

Lingqian Wu,

Central South University, China

Reviewed by:

Lei Wang,

Fudan University, China

Baoheng Gui,

The Second Affiliated Hospital of Guangxi Medical University, China

*Correspondence:

Ling Sun

sunling6299@163.com

Xin-Yu Guo

iris92@126.com

Specialty section:

This article was submitted to

Genetics of Common and Rare

Diseases,

a section of the journal

Frontiers in Genetics

Received: 18 August 2021

Accepted: 25 October 2021

Published: 19 November 2021

Citation:

Chen J, Xu J, Chen Z-H, Yin M-N, Guo $X-Y$ and Sun $L$ (2021) Case Report: Identification of Germline

Chimerism in Monochorionic

Dizygotic Twins.

Front. Genet. 12:744890

doi: 10.3389/fgene.2021.744890

\section{Case Report: Identification of Germline Chimerism in Monochorionic Dizygotic Twins}

\author{
Juan Chen ${ }^{1}$, Jian Xu ${ }^{1}$, Zhi-Heng Chen ${ }^{1}$, Min-Na Yin ${ }^{1}$, Xin-Yu Guo ${ }^{2 *}$ and Ling Sun ${ }^{1 *}$ \\ ${ }^{1}$ Department of Assisted Reproductive technology, Guangzhou Women and Children's Medical Center, Guangzhou Medical \\ University, Guangzhou, China, ${ }^{2}$ Reproductive Medicine Center, Department of Obstetrics and Gynecology, Guangzhou General \\ Hospital of Guangzhou Military Region, Guangzhou, China
}

Monochorionic twins are generally considered to be monozygotic, as monochorionic dizygotic (MCDZ) twins are extremely rare in natural pregnancies. Several studies have reported this rare occurrence, and most of these pregnancies have been conceived by assisted reproductive technology (ART). These reports mostly focused on MCDZ twin pregnancies and the childhood development of the twins; a follow-up into adulthood and the effect on their reproduction has not been reported. In this case study, we report a case of chimerism in opposite-sex MCDZ twins who were naturally conceived and have reached reproductive maturity. We collected oral mucosal, endometrial, and germ cells from the twins and evaluated their chimerism using single-nucleotide polymorphism (SNP) array and droplet digital PCR (ddPCR). The SNP array showed that they had 4,049 non-allele shared loci, and they inherited nearly 50\% informative SNP loci from each parent, confirming that they are dizygotic twins. We found that the female twin had a 46, XX (2)/46, XY (78) karyotype in her peripheral blood. The SNP array confirmed that the female twin and male twin had the same blood haplotype. The ddPCR result showed 92.84 ( \pm $1.80 \%$ ) chimerism in her blood. In case of chimerism in her germline, the female twin chose preimplantation genetic testing for aneuploidy for her blastocysts. Fortunately, the patient only had blood chimerism. A healthy boy was born at 39 weeks of gestation.

Keywords: MCDZ twins, tissues chimerism, germ cell, PGT-A, ddPCR

\section{INTRODUCTION}

Monochorionic twins are generally considered to be monozygotic. However, monochorionic dizygotic (MCDZ) twins, an extremely rare occurrence, have been reported due to rapidly improving cytogenetic and DNA sequencing techniques (Nylander and Osunkoya, 1970; Lee et al., 2014a; Rodriguez-Buritica et al., 2015; Korsun et al., 2016; Uysal et al., 2018; Gabbett et al., 2019). MCDZ twins share the same placenta, which leads to cell exchange between embryos with different genotypes via vascular anastomoses, resulting in blood chimerism. In theory, the cell exchange between two fetuses only occurs in blood cells. However, chimerism in other tissues has been reported in several studies (Fumoto et al., 2014; Rodriguez-Buritica et al., 2015; Korsun et al., 2016; Uysal et al., 2018), and the early-stage fusion of dizygotic embryos was speculated to be the reason for occurrence. The presence of tissue chimerism might lead to serious clinical consequences in MCDZ twins, especially in opposite-sex MCDZ twins. When chimerism occurs, the karyotype and genotype in the blood cannot present other tissue status. In 2003, Johannsen et al. 

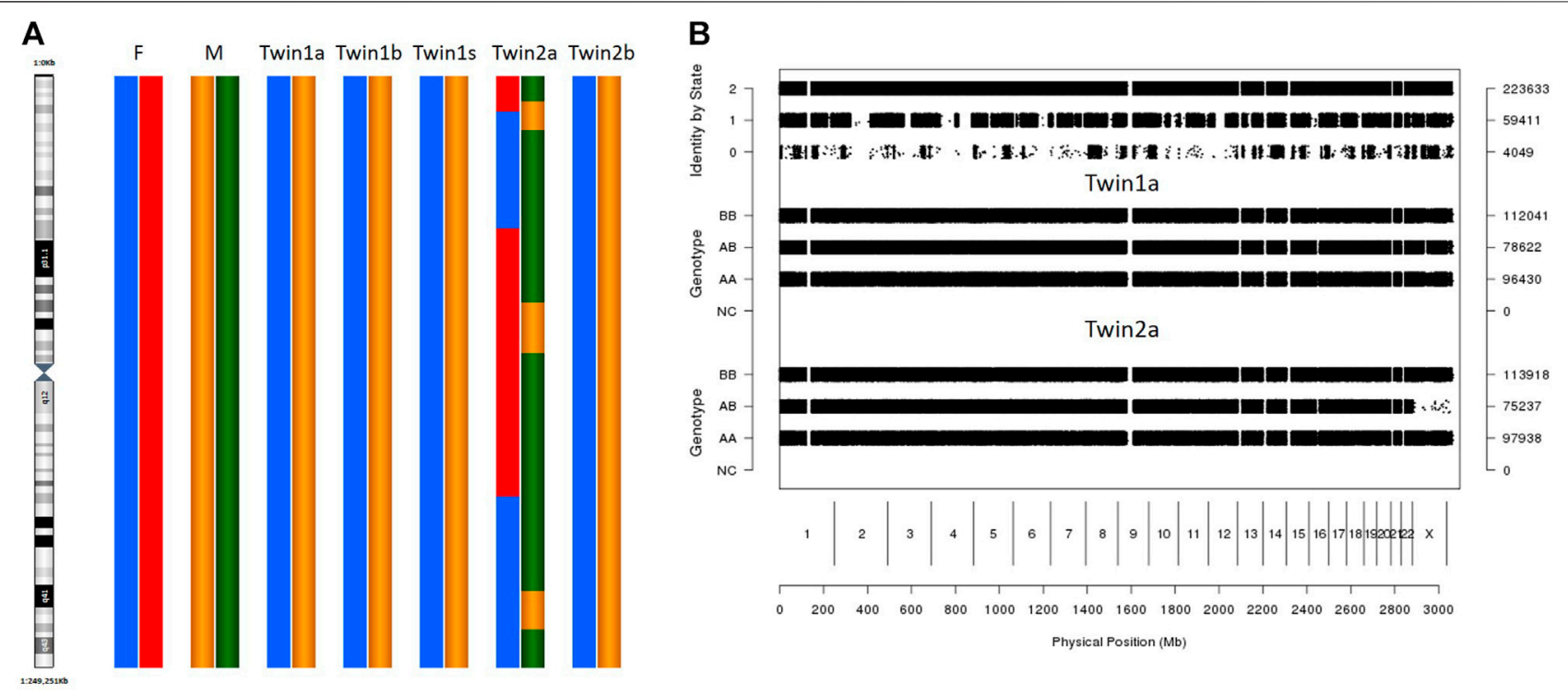

FIGURE 1 I SNP array result of different tissues. (A) Karyomapping (chr1) of different tissues in twins. F: father M: mother. Twin1a/b/s: Oral mucosa cells, blood, and sperm in twin1. Twin2 a/b: Oral mucosa cells, blood in twin2. (B) Result of pairwise SNP analysis in genome wide. SNP analysis used the SNPduo Web tool. Identity by state (IBS) 2: both alleles shared between twins; IBS1: single allele shared between twins; IBS0: non-allele shared. Twin1a: Oral mucosa cells twin1. Twin2 a: Oral mucosa cells in twin2.

(2003) reported a case of erroneous sex classification that led to ovary removal in a twin girl since she presented with a $46, \mathrm{XY}$ blood karyotype and was suspected of having gonadal dysgenesis. In addition, when blood chimerism occurs, it might lead to an incorrect interpretation of blood group phenotypes in blood donors (Bluth et al., 2007), increasing the risk of a hemolytic reaction in recipients (Parva et al., 2009; Assaf et al., 2010). Most reports focus on MCDZ twin pregnancies, and they follow up on their childhood development for a few years. A report on adult MCDZ twins and the effect on their reproduction is rare, as most of the reported cases were recently conceived by assisted reproductive technology (ART). Here, we present naturally conceived, opposite-sex MCDZ twins who reached reproductive maturity; we investigated their chimerism in different tissues and germline cells. This information will assist researchers and clinicians in evaluating the clinical consequences of chimerism and raising awareness on the condition.

\section{CASE DESCRIPTION}

A 27-year-old woman (twin 2) who miscarried once in 2018 visited our center to consult for infertility. Her physical examination showed normal breast and internal and external genitalia development. She had menarche at 14 years of age, and her menstruation cycles were regular. Basal hormone levels and anti-Müllerian hormone (AMH; $5.05 \mathrm{ng} / \mathrm{ml}$ ) levels were normal. A karyotype analysis of her peripheral blood showed a 46, XX (2)/ 46, XY (78) chimeric karyotype. She claimed that she has never received a blood transfusion before, but she does have a twin brother (twin 1). Her mother informed us that she had one large
TABLE 1 | ddPCR result of different tissue in twin 1 and twin 2.

\begin{tabular}{llcc} 
Tissue & & Twin 1 (\%) & Twin 2 (\%) \\
\hline Twin1 & Blood & $100.29 \pm 1.57$ & $-0.29 \pm 1.57$ \\
& Oral mucosa & $99.3 \pm 13.33$ & $0.7 \pm 13.33$ \\
& Sperm & $100.76 \pm 2.51$ & $-0.76 \pm 2.51$ \\
Twin2 & Blood & $92.84 \pm 1.80$ & $7.16 \pm 1.80$ \\
& Oral mucosa & $0.49 \pm 1.83$ & $99.51 \pm 1.83$ \\
& Endometrium $^{*}$ & $-0.09 \pm 1.16$ & $100.09 \pm 1.16$ \\
& Oocyte 1 $^{\mathrm{a}}$ & $1.18 \pm 3.18$ & $98.82 \pm 3.18$ \\
& Oocyte 2 $^{\mathrm{a}}$ & $0.77 \pm 2.19$ & $99.23 \pm 2.19$
\end{tabular}

All data are presented as the mean $\pm S D$.

${ }^{a}$ Only 11 sets of Twin 2 informative SNP probes were used for oocytes.

placenta when she gave birth to the twins. We assumed that the patient's chimerism came from her twin brother and we informed her of her blood chimerism and germline chimerism risk. After informed consent was obtained, we collected buccal cells and blood from the twins. Additionally, sperm cells were collected from her twin brother. DNA was extracted and a singlenucleotide polymorphism (SNP) array (Karyomapping, Illumina) was performed.

The karyomapping results showed that twin 2 had different genetic karyotypes between her oral mucosal cells and blood cells (Figure 1A), and her blood karyotype was identical to that of twin 1. The karyomapping results also indicated which alleles are shared by the twins (Figure 1B). They have 4,049 non-alleleshared loci, which means that they were conceived by different oocytes and sperms. They inherited nearly 50\% informative SNP loci from each parent (data not shown), confirming that they are dizygotic twins. 
Based on the karyomapping results, we designed 23 pairs of ddPCR primers (Supplementary Table S1) to distinguish chimerism in different tissues (oral mucosa, endometrium, and germline cells). The results indicated that twin 1 has the same DNA in different tissues, and twin 2 has two sets of DNA in her blood with $92.84 \pm 1.80 \%$ chimerism (Table 1). There was no chimerism detected in the oral mucosa or endometrium of twin 2 (Table 1).

In order to determine chimerism in germline cells, the patient chose preimplantation genetic testing for aneuploidy on her blastocysts. Thirty oocytes were retrieved from the ovaries of the patient, followed by fertilization via an intracytoplasmic sperm injection (ICSI). Twenty-one oocytes were successfully fertilized, and the zygotes were cultured in sequential G1/G2 media for 5-6 days. Ten blastocysts were obtained, and a biopsy of 5-10 cells from trophectoderm (TE) tissue was performed on day 5 or day 6 , depending on the blastocyst stage. To confirm germline chimerism, two oocytes from failed fertilization and endometrial cells were collected. To obtain sufficient DNA for subsequent studies, whole genome amplification was performed on the TE biopsy and oocytes. Thereafter, the amplified DNA was dispensed onto Human CytoSNP-12 DNA BeadChips (Illumina, San Diego, CA, United States). Ten blastocysts and two oocytes were assessed for informative SNPs that were present in twin 1 but not in twin 2. Approximately, 0-5 out of the 300,000 SNPs were found in each blastocyst (data not shown). This result did not support the existence of chimerism in blastocysts and oocytes.

The SNP array was also analyzed for preimplantation genetic testing for aneuploidy testing. The results showed that seven embryos were euploid, one was aneuploid, and two were mosaic. A single euploid blastocyst was selected and transferred. At 12 weeks of gestation, non-invasive prenatal testing was performed and no aneuploidy was detected. A healthy boy was born at 39 weeks of gestation.

\section{DISCUSSION}

Several studies have reported chimerism in tissues other than the blood (Fumoto et al., 2014; Rodriguez-Buritica et al., 2015; Korsun et al., 2016; Uysal et al., 2018). However, in this case, chimerism was only observed in blood cells and not in other tissues. We assessed cervical epithelial cells from twin 2, and the initial ddPCR result showed the same karyotype as identified in her twin brother, indicating potential tissue chimerism. However, in the repeated experiment, we used flow cytometry to exclude leukocytes by staining with the antiCD45 antibody, and it was found that most cells were $\mathrm{CD}_{4} 5^{+}$. This means that most of the cervical epithelium cells we initially collected were leukocytes. Unfortunately, after cell sorting, the remainder $\mathrm{CD} 45^{-}$cells were not sufficient for further study.

In previous studies, there are two hypotheses to explain the mechanism of tissue chimerism, other than blood. One theory is that the fusion of dizygotic embryos at an early stage causes tissue chimerism. However, this theory is not supported by the developmental process of embryos. According to the embryology theory, the zygote is enclosed within the zona pellucida for at least 5 days until it develops into a blastocyst and hatches from the zona pellucida. Thus, the dizygotic embryo could not fuse until day five of embryogenesis. The blastocyst then divides into inner cell mass (fetal development) and trophectoderm (placental development). It is therefore unlikely that the inner mass of the blastocyst fuses and divides into two embryos. Another hypothesis is that tissue chimerism originates from bone marrow stem cells. The observation of bone marrow transplant patients led to this hypothesis. A study reported that after a female patient received a bone marrow transplant from a male donor (Taylor, 2004; Liu et al., 2018), the Y chromosome was detected in her endometrial cells. As a result, the author speculated that bone marrow stem cells are involved in endometrial regeneration. However, in our case this theory could not explain why, after numerous menstruation cycles, the endometrium of twin 2 never presented chimerism. As blood-cell contamination can occur in most tissue collections, the conclusion of tissue chimerism in MCDZ twins should be drawn cautiously.

An increasing number of MCDZ twins has been reported, and at least $80 \%$ of the reported cases were conceived by ART (Williams et al., 2004; Aoki et al., 2006; Ekelund et al., 2008; Parva et al., 2009; Assaf et al., 2010; Choi et al., 2013; Smeets et al., 2013; Lee et al., 2014a; Lee et al., 2014b; Fumoto et al., 2014; Korsun et al., 2016; Mayeur Le Bras et al., 2016). This indicates that when monochorionic twins, which originated from the transfer of two embryos, are diagnosed by ultrasound, we should consider two possibilities: the first possibility is that one embryo died and the other embryo developed to be monochorionic monozygotic (MCMZ), or alternatively, both embryos survive, share the placenta, and develop into MCDZ. Although MCDZ twinning is rare, this occurrence is expected to increase with the development of ART. Since same-sex MCDZ twinning is easily overlooked, the MCDZ incidence rate could be higher than previously reported. In order to avoid iatrogenic complications caused by chimerism, monochorionic twins originating from the transfer of two embryos should be recommended for the SNP array or other chromosomal screenings to exclude the possibility of blood chimerism.

In this study, we have gained some insight into MCDZ twins; however, there were limitations within this study. We only examined three different kinds of tissues and blood, and therefore, we could not rule out the possibility of chimerism in other tissues. Besides performing non-invasive prenatal testing, we did not test for tissue chimerism in the offspring of the patient. We will follow up on his condition in a subsequent study.

\section{DATA AVAILABILITY STATEMENT}

The original contributions presented in the study are included in the article/Supplementary Material; further inquiries can be directed to the corresponding authors. 


\section{ETHICS STATEMENT}

The studies involving human participants were reviewed and approved by the Ethics Committee of Guangzhou Women and Children Medical Center. The patients/participants provided their written informed consent to participate in this study.

\section{AUTHOR CONTRIBUTIONS}

JC and Z-HC performed experiments, analyzed the data, prepared tables and figures, and wrote the manuscript. JX and $\mathrm{M}-\mathrm{NY}$ collected the clinical information and follow up the patients. LS and X-YG designed the study, supervised the

\section{REFERENCES}

Aoki, R., Honma, Y., Yada, Y., Momoi, M. Y., and Iwamoto, S. (2006). Blood Chimerism in Monochorionic Twins Conceived by Induced Ovulation: Case Report. Hum. Reprod. 21, 735-737. doi:10.1093/humrep/dei379

Assaf, S. A., Randolph, L. M., Benirschke, K., Wu, S., Samadi, R., and Chmait, R. H. (2010). Discordant Blood Chimerism in Dizygotic Monochorionic LaserTreated Twin-Twin Transfusion Syndrome. Obstet. Gynecol. 116 Suppl 2 (Suppl. 2), 483-485. doi:10.1097/AOG.0b013e3181e4bec7

Bluth, M. H., Reid, M. E., and Manny, N. (2007). Chimerism in the Immunohematology Laboratory in the Molecular Biology Era. Transfus. Med. Rev. 21, 134-146. doi:10.1016/j.tmrv.2006.11.002

Choi, D.-H., Kwon, H., Lee, S. D., Moon, M.-J., Yoo, E.-G., Lee, K.-H., et al. (2013). Testicular Hypoplasia in Monochorionic Dizygous Twin with Confined Blood Chimerism. J. Assist Reprod. Genet. 30, 1487-1491. doi:10.1007/s10815-0130109-8

Ekelund, C. K., Skibsted, L., Søgaard, K., Main, K. M., Dziegiel, M. H., Schwartz, M., et al. (2008). Dizygotic Monochorionic Twin Pregnancy Conceived Following Intracytoplasmic Sperm Injection Treatment and Complicated by Twin-Twin Transfusion Syndrome and Blood Chimerism. Ultrasound Obstet. Gynecol. 32, 832-834. doi:10.1002/uog.6221

Fumoto, S., Hosoi, K., Ohnishi, H., Hoshina, H., Yan, K., Saji, H., et al. (2014). Chimerism of Buccal Membrane Cells in a Monochorionic Dizygotic Twin. Pediatrics 133, e1097-e1100. doi:10.1542/peds.2013-1938

Gabbett, M. T., Laporte, J., Sekar, R., Nandini, A., McGrath, P., Sapkota, Y., et al. (2019). Molecular Support for Heterogonesis Resulting in Sesquizygotic Twinning. N. Engl. J. Med. 380, 842-849. doi:10.1056/nejmoa1701313

Johannsen, T. H., Lundsteen, C., Visfeldt, J., Schwartz, M., Petersen, B. L., Byskov, A. G., et al. (2003). Erroneous Genetic Sex Determination of a Newborn Twin Girl Due to Chimerism Caused by Foetal Blood Transfusion. Horm. Res. Paediatr. 60, 148-151. doi:10.1159/000072527

Korsun, P., Bals-Pratsch, M., Ortmann, O., Markus, S., and Germer, U. (2016). Antenatal Diagnosis of Dizygotic, Monochorionic Twins Following IVF/ICSI. Geburtshilfe Frauenheilkd 76, 727-730. doi:10.1055/s-0042-101024

Lee, H. J., Yoon, S. C., Ko, J. M., Seong, M. W., Park, S. S., Choi, J. S., et al. (2014). Monochorionic Dizygotic Twins with Discordant Sex and Confined Blood Chimerism. Eur. J. Pediatr. 173, 1249-1252. doi:10.1007/s00431-014-2312-8

Lee, O.-J., Cho, D., Shin, M.-G., Kim, S.-O., Park, J.-T., Kim, H. K., et al. (2014). The First Known Case of Blood Group Chimerism in Monochorionic Dizygotic Twins in Korea. Ann. Lab. Med. 34, 259-262. doi:10.3343/alm.2014.34.3.259

Liu, Y., Tal, R., Pluchino, N., Mamillapalli, R., and Taylor, H. S. (2018). Systemic Administration of Bone Marrow-Derived Cells Leads to Better Uterine Engraftment Than Use of Uterine-Derived Cells or Local Injection. J. Cel. Mol. Med. 22, 67-76. doi:10.1111/jcmm.13294 interpretation, statistical analysis of the data, and edited the manuscript.

\section{FUNDING}

This work was supported by the National Natural Science Foundation of China (81800110).

\section{SUPPLEMENTARY MATERIAL}

The Supplementary Material for this article can be found online at: https://www.frontiersin.org/articles/10.3389/fgene.2021.744890/ full\#supplementary-material

Mayeur Le Bras, A., Petit, F., Benachi, A., Bedel, B., Oucherif, S., Martinovic, J., et al. (2016). Confined Blood Chimerism in a Monochorionic Dizygotic Sex Discordant Twin Pregnancy Conceived after Induced Ovulation. Birth Defects Res. A: Clin. Mol. Teratology 106, 298-303. doi:10.1002/bdra.23457

Nylander, P. P., and Osunkoya, B. O. (1970). Unusual Monochorionic Placentation with Heterosexual Twins. Obstet. Gynecol. 36, 621-625.

Parva, M., Donnenfeld, A. E., and Gerson, A. (2009). Trizygotic Dichorionic Triplets with 46,XX/46,XY Chimerism in Both Fetuses of the Monochorionic Pair. Prenat. Diagn. 29, 1091-1093. doi:10.1002/pd.2368

Rodriguez-Buritica, D., Rojnueangnit, K., Messiaen, L. M., Mikhail, F. M., and Robin, N. H. (2015). Sex-discordant Monochorionic Twins with Blood and Tissue Chimerism. Am. J. Med. Genet. 167, 872-877. doi:10.1002/ajmg.a.37022

Smeets, D., van Vugt, J. M. G., Gomes, I., van den Heuvel, S., van Heijst, A., Reuss, A., et al. (2013). Monochorionic Dizygous Twins Presenting with Blood Chimerism and Discordant Sex. Twin Res. Hum. Genet. 16, 799-801. doi:10.1017/thg.2013.41

Taylor, H. S. (2004). Endometrial Cells Derived from Donor Stem Cells in Bone Marrow Transplant Recipients. JAMA 292, 81-85. doi:10.1001/jama.292.1.81

Uysal, N. Ş., Gülümser, Ç., Çelik, Z. Y., Zeyneloğlu, H. B., and Yanık, F. F. B. (2018). Fetal Sex Discordance in a Monochorionic Twin Pregnancy Following Intracytoplasmic Sperm Injection: A Case Report of Chimerism and Review of the Literature. J. Obstet. Gynaecol. Res. 44, 576-582. doi:10.1111/jog.13514

Williams, C. A., Wallace, M. R., Drury, K. C., Kipersztok, S., Edwards, R. K., Williams, R. S., et al. (2004). Blood Lymphocyte Chimerism Associated with IVF and Monochorionic Dizygous Twinning: Case Report. Hum. Reprod. 19, 2816-2821. doi:10.1093/humrep/deh533

Conflict of Interest: The authors declare that the research was conducted in the absence of any commercial or financial relationships that could be construed as a potential conflict of interest.

Publisher's Note: All claims expressed in this article are solely those of the authors and do not necessarily represent those of their affiliated organizations, or those of the publisher, the editors, and the reviewers. Any product that may be evaluated in this article, or claim that may be made by its manufacturer, is not guaranteed or endorsed by the publisher.

Copyright (c) 2021 Chen, Xu, Chen, Yin, Guo and Sun. This is an open-access article distributed under the terms of the Creative Commons Attribution License (CC BY). The use, distribution or reproduction in other forums is permitted, provided the original author(s) and the copyright owner(s) are credited and that the original publication in this journal is cited, in accordance with accepted academic practice. No use, distribution or reproduction is permitted which does not comply with these terms. 